\title{
Pregnancy with Intrauterine Device
}

National Cancer Institute

\section{Source}

National Cancer Institute. Pregnancy with Intrauterine Device. NCI Thesaurus. Code C111950.

Co-existence of an intrauterine pregnancy with an intrauterine device. 